\title{
On the location of public bads: strategy-proofness under two-dimensional single-dipped preferences
}

Citation for published version (APA):

Öztürk, M., Peters, H. J. M., \& Storcken, A. J. A. (2012). On the location of public bads: strategyproofness under two-dimensional single-dipped preferences. METEOR, Maastricht University School of Business and Economics. METEOR Research Memorandum No. 040 https://doi.org/10.26481/umamet.2012040

Document status and date:

Published: 01/01/2012

DOI:

10.26481/umamet.2012040

Document Version:

Publisher's PDF, also known as Version of record

\section{Please check the document version of this publication:}

- A submitted manuscript is the version of the article upon submission and before peer-review. There can be important differences between the submitted version and the official published version of record. People interested in the research are advised to contact the author for the final version of the publication, or visit the DOI to the publisher's website.

- The final author version and the galley proof are versions of the publication after peer review.

- The final published version features the final layout of the paper including the volume, issue and page numbers.

Link to publication

\footnotetext{
General rights rights.

- You may freely distribute the URL identifying the publication in the public portal. please follow below link for the End User Agreement:

www.umlib.nl/taverne-license

Take down policy

If you believe that this document breaches copyright please contact us at:

repository@maastrichtuniversity.nl

providing details and we will investigate your claim.
}

Copyright and moral rights for the publications made accessible in the public portal are retained by the authors and/or other copyright owners and it is a condition of accessing publications that users recognise and abide by the legal requirements associated with these

- Users may download and print one copy of any publication from the public portal for the purpose of private study or research.

- You may not further distribute the material or use it for any profit-making activity or commercial gain

If the publication is distributed under the terms of Article $25 \mathrm{fa}$ of the Dutch Copyright Act, indicated by the "Taverne" license above, 
Murat Öztürk, Hans Peters, Ton Storcken

On the location of public bads: strategy-proofness under twodimensional single-dipped preferences

RM/ 12/040

\section{METEOR}

Maastricht University School of Business and Economics

Maastricht Research School of Economics

of Technology and Organization

\section{PO. Box 616}

NL - 6200 MD Maastricht

The Netherlands 


\title{
On the location of public bads: strategy-proofness under two-dimensional single-dipped preferences
}

\author{
Murat Öztürk* Hans Peters* Ton Storcken*
}

July, 2012

\begin{abstract}
In a model with finitely many agents who have single-dipped Euclidean preferences on a polytope in the Euclidean plane, a rule assigns to each profile of reported dips a point of the polytope. A single-best point is a point which is the unique point at maximal distance from some other point of the polytope. It is proved that any strategy-proof and Pareto optimal rule is a dictatorship unless the polytope has exactly two singlebest points or it has exactly four single-best points which form the vertices of a rectangle. In the latter cases strategy-proof and Pareto optimal rules can be obtained by committee voting (simple games) between the singlebest alternatives. This framework models situations where public bads such as garbage dumping grounds or nuclear plants have to be located within a confined region.
\end{abstract}

JEL Classification: D71

Keywords: Single-dipped preferences, strategy-proofness, committee voting

\section{Introduction}

Consider the problem of locating a windmill park, garbage dumping ground, heavy industry, or nuclear plant within a confined area, such as a city, province, or country. These are examples of public bads: people agree on their usefulness or even necessity but typically do not want them in their backyards. In this paper we assume that the public bad is to be located within a given region - a subset of the plane - and that the location is determined by voting among a set of agents (for instance, inhabitants of the region, or political representatives). Each agent is characterized by a Euclidean single-dipped preference: there is a worst point, the dip, which is a specific point of the region, and preference increases with the Euclidean distance from this dip. A vote then consists of

\footnotetext{
*Maastricht University, Department of Quantitative Economics, P.O. Box 616, 6200 MD Maastricht, The Netherlands. Email addresses: M.Ozturk@maastrichtuniversity.nl, H.Peters@maastrichtuniversity.nl, T.Storcken@maastrichtuniversity.nl.
} 
reporting one's dip. Typically, these dips are private knowledge: an agent's dip could coincide with his residence, the school of his children, an important natural resource, etc. In order for the location of the public bad to be based on the right information about the agents' preferences the (voting) rule should be strategy-proof: no agent should be able to achieve a location farther away from his true dip by not reporting truthfully. Also, we will require the rule to be Pareto optimal: it should not assign a location for which there is another location at least as distant for all and strictly farther away for some agents, given the reported dips.

In this paper we assume that the region is a two-dimensional polytope $A$, i.e., the convex hull of at least three but finitely many points, which are the extreme points of the polytope. An important concept is that of a single-best point, i.e., a point of $A$ that is the unique point at maximal distance from some other point of $A$. Single-best points are always extreme points, although not every extreme point is necessarily a single-best point. For instance, if the boundary of $A$ is a triangle with vertices $a, b$, and $c$, then these three points are the single-best points if the triangle is acute. However, if the angle at $a$ is right then $a$ can be a best point but never a single-best point, and if this angle is obtuse then $a$ is not even a best point.

We assume that there are $n \geq 2$ agents; a profile is a vector of $n$ points of $A-$ interpreted as a vector of reported dips - and a rule assigns a point of $A$ to each profile. Our main results are as follows. If $A$ does not have exactly two singlebest points or if $A$ does not have exactly four single-best points which form the vertices of a rectangle, then a strategy-proof and Pareto optimal rule must be dictatorial: there is a fixed agent $d$ such that the rule always assigns a point at maximal distance from $d$ 's dip. This result is obtained by proving that the set of decisive coalitions is an ultrafilter and, in particular, closed under taking intersections. The last property does not hold if $A$ has exactly two single-best points or exactly four single-best points which are the vertices of a rectangle. For the case that $A$ has exactly two single-best points $a$ and $b$-which means, roughly, that $A$ is sufficiently flat with $a$ and $b$ as 'end points' - we characterize all strategy-proof and Pareto optimal rules under a few mild additional (tiebreaking) assumptions: such rules are described by committee (simple game) voting between $a$ and $b$. Similarly, for the case where $A$ has exactly four singlebest points which are the vertices of a rectangle, we can obtain strategy-proof and Pareto optimal rules by committee voting between the upper and lower and between the left and right vertices of the rectangle; for this case we content ourselves by giving an example.

The message of the paper is therefore that dictatorship can be avoided if the region is sufficiently flat (in terms of width) or if we can identify four locations which are the vertices of a rectangle and which are the only single-best points.

Since some parts of the proof rely on the finiteness of the set of extreme points of a region $A$, extension of our results to general compact convex set does not seem straightforward. In a companion note (Öztürk, Peters, and Storcken, 2012) we show that dictatorship continues to hold when $A$ is a disc (a circle and its inside) but this needs a separate proof. 
Related literature To the dictatorship part of our results the classical work of Gibbard (1973) and Satterthwaite (1975) does not directly apply since we do not have full preference domains. An exception is the acute triangle: if we know that the rule assigns a vertex then in this case dictatorship follows from Gibbard-Satterthwaite since single-dipped preferences generate the full domain of six (strict) preferences over the vertices (see the end of Section 3).

Although single-dipped and single-peaked preferences are both special cases of value restrictions (Inada, 1964) their consequences in spatial models like ours seem to be quite different. In particular, under single-dipped preferences Pareto optimality of a rule, but also weaker conditions such as unanimity, force the outcome of the rule to be on the boundary of the region under consideration which is also why the restriction to compact sets is natural in the present framework. As a result, under single-dipped preferences much is going to depend on the shape of the boundary of the region. Generally speaking, single-peakedness of preferences seems to allow for more possibilities and to lead less frequently to dictatorship (Black, 1948; Moulin, 1980; Border and Jordan, 1983).

In spite of its apparent relevance, existing work on collective decision making in the presence of single-dipped preferences is relatively limited. As far as we know ours is the first paper to study strategy-proofness under single-dipped preferences in two-dimensional space. The one-dimensional case has been studied before. Peremans and Storcken (1999) characterize all strategy-proof rules for the case that $A$ is an arbitrary subset of the real line and preferences are single-dipped (and not necessarily symmetric). An implication of their work is that individual and coalitional strategy-proofness are equivalent in this model. Also Barberà, Berga, and Moreno (2009) reach this conclusion and sharpen the bounds on the range of strategy-proof rules, depending on different subclasses of single-dipped preferences. Manjunath (2009) specifically studies unanimous and strategy-proof rules defined on a real interval. Thus, all these works concern the location of a public bad where the region $A$ is one-dimensional.

The private good case with single-dipped preferences is considered in Klaus, Peters, and Storcken (1997), which deals with the division of a perfectly divisible commodity. Klaus (2001) considers the case where this commodity is an indivisible object and has to be allocated to one of the agents. Ehlers (2002) considers the probabilistic allocation of an indivisible object.

The proof of the dictatorship result based on decisive coalitions and ultrafilters has been used before, see Kirman and Sondermann (1972) and Hansson (1976).

Finally, there is a literature on mechanism design for the location of public bads when monetary transfers are allowed, see for instance Kunreuther and Kleindorfer (1986) or, more recently, Lescop (2007) or Besfamille and Lozachmeur (2010).

Organization of the paper Section 2 starts with notations, the basic model, and some preliminary results. In Section 3 we derive the dictatorship result, and in Section 4 we consider the non-dictatorship cases. Section 5 concludes. 


\section{Notations, basic model, and preliminaries}

We start by fixing some notations to be used throughout the paper.

\section{$2.1 \quad$ Notations}

For a subset $X$ of $\mathbb{R}^{2}$ (endowed with the usual topology) we denote by $\partial X$ its boundary, by $X^{\circ}$ its interior, and by $\bar{X}$ its closure.

Let $a, b, c \in \mathbb{R}^{2}$ be three different points. Then $[a, b]$ denotes the closed line segment between points $a$ and $b,] a, b$ [ the (relatively) open line segment between $a$ and $b$, and $[a, b[$ and $] a, b]$ the two half open line segments. The midpoint of $[a, b]$ is denoted by $m_{a, b}$ and its perpendicular bisector by $\ell_{a, b}$. For $x \in \mathbb{R}^{2}$ we denote by $\sigma_{a, b}(x)$ the reflection of $x$ in the line $\ell_{a, b}$, i.e., $\ell_{a, b}$ is the perpendicular bisector of $\left[x, \sigma_{a, b}(x)\right]$.

If $a, b$, and $c$ are non-collinear we denote $m_{a, b, c}=\ell_{a, b} \cap \ell_{b, c} \cap \ell_{a, c}$ (and regard $m_{a, b, c}$ as a point rather than a set). Further, $\ell(a, b)$ denotes the straight line through the points $a$ and $b$.

The Euclidean distance between $a$ and $b$ is denoted by $\|a-b\|$. A straight line $\ell$ divides the plane $\mathbb{R}^{2}$ into two half-planes. For $a \notin \ell, H(\ell, a)$ indicates the closed half-plane containing $a$; we write $H^{\circ}(\ell, a)$ for the open half-plane, thus $H^{\circ}(\ell, a)=H(\ell, a)^{\circ}$. The circle with center $a$ and radius $r \geq 0$ is denoted by $\odot(a, r)$, hence $\odot(a, r)=\left\{x \in \mathbb{R}^{2}:\|a-x\|=r\right\}$. For $x, y \in \mathbb{R}^{2}$ on the same circle with center $a \in \mathbb{R}^{2}$, we write $[a ; x \frown y]$ for the arc between $x$ and $y$, that is, $[a ; x \frown y]=\odot(a,\|a-x\|) \backslash H^{\circ}(\ell(x, y), a)$ if $a$ is not on $\ell(x, y)$, otherwise $[a ; x \frown y]=\odot(a,\|a-x\|)$.

For an arbitrary set $D$ we denote by $|D|$ its cardinality.

\subsection{The basic model}

We next formulate the basic model. There is a finite set $N=\{1, \ldots, n\}(n \geq 2)$ of agents and a compact subset $A \subseteq \mathbb{R}^{2}$ of alternatives. Each agent is endowed with a (Euclidean single-dipped) preference $R_{x}, x \in A$, defined as follows: for all $y, z \in A,(y, z) \in R_{x}$ if $\|x-y\| \geq\|x-z\|$. The alternative $x$ is the dip of preference $R_{x}$. Clearly, preference $R_{x}$ is completely determined by its dip; therefore, if convenient, we will denote a preference by its dip. An element $p=(p(1), \ldots, p(n)) \in A^{N}$, where $p(i)$ is the dip of agent $i$, is called a profile (of single-dipped preferences). A social choice function or simply rule is a map $\varphi: A^{N} \rightarrow A$. We are interested in rules that satisfy the following two properties. Rule $\varphi$ is called

[PO] Pareto optimal if for every profile $p \in A^{N}$, every agent $i \in N$, and every alternative $a \in A$ with $\|p(i)-a\|>\|p(i)-\varphi(p)\|$ there is an agent $j \in N$ such that $\|p(j)-a\|<\|p(j)-\varphi(p)\|$;

[SP] strategy-proof if for every agent $i$ in $N$ and all profiles $p, q \in A^{N}$, with $q(j)=p(j)$ for all $j \in N \backslash\{i\}$, we have $\|p(i)-\varphi(p)\| \geq\|p(i)-\varphi(q)\|$. 
The interpretation of Pareto optimality is as usual, and strategy-proofness (also as usual) implies that no agent can improve the outcome by misreporting his preference.

A subset of $N$ is called a coalition. As the reader may verify, strategyproofness is equivalent to the following condition. Rule $\varphi$ is called

[ISP] intermediate strategy-proof if for every coalition $S \subseteq N$ and all profiles $p, q \in A^{N}$ such that $p(j)=q(j)$ for all $j \in N \backslash S$ and there is an alternative $a$ with $p(i)=a$ for all $i \in S$, we have $\|a-\varphi(p)\| \geq\|a-\varphi(q)\|$.

Often ISP will be used instead of SP without explicit mentioning.

\subsection{Preliminaries}

Let $S \subseteq N$ be a coalition and let $a, b \in A$. By $\left(a^{S}, b^{N \backslash S}\right) \in A^{N}$ we denote the profile $p$ with $p(i)=a$ for all $i \in S$ and $p(i)=b$ for all $i \in N \backslash S$. (If $S=N$ we usually write $a^{N}$ instead of $\left(a^{N}, b^{\emptyset}\right)$.) To such a two-dip profile a Pareto optimal rule always assigns a boundary point, as the following lemma shows.

Lemma 2.1 Let $a, b \in A, S \subseteq N$, and $p=\left(a^{S}, b^{N \backslash S}\right)$. Let $\varphi: A^{N} \rightarrow A$ be a Pareto optimal rule. Then $\varphi(p) \in \partial A$.

Proof. Let $\ell=\ell(a, b)$ if $a \neq b$ and let $\ell$ be an arbitrary straight line through $a$ if $a=b$. Let $x$ be the intersection of $\ell$ and the line $\ell^{\prime}$ through $\varphi(p)$ perpendicular to $\ell$. Now for points $c$ on $\ell^{\prime}$ such that $\left.\left.\varphi(p) \in\right] c, x\right]$, it follows that $\|a-c\|>$ $\|a-\varphi(p)\|$ and $\|b-c\|>\|b-\varphi(p)\|$. Hence, by Pareto optimality we must have $c \notin A$, so that $\varphi(p)$ cannot be an interior point of $A$. Hence $\varphi(p) \in \partial A$.

Observe that the proof of this lemma does not use the compactness of the set $A$. Thus, the lemma justifies our restriction to compact sets.

For a preference $R_{a}$, the set of best alternatives is denoted by $\mathfrak{b}\left(R_{a}\right)$ or by $\mathfrak{b}(a)$, i.e., $\mathfrak{b}\left(R_{a}\right)=\mathfrak{b}(a)=\{y \in A:\|a-y\| \geq\|a-z\|$ for all $z \in A\}$. A coalition $S \subseteq N$ is decisive (given a rule $\varphi$ ) if $\varphi(p) \in \mathfrak{b}\left(R_{a}\right)$ for all $a \in A$ and all $p \in A^{N}$ with $p(i)=a$ for all $i \in S$. Clearly, if $S$ is decisive and $S \subseteq T \subseteq N$, then $T$ is decisive. A rule $\varphi$ is dictatorial if there is a dictator, i.e., an agent $d \in N$ such that $\{d\}$ is decisive.

A collection $\mathcal{W}$ of subsets of $N$ is called an ultrafilter on $N$ if for all subsets $S, T \subseteq N$ we have (i) $\emptyset \notin \mathcal{W}$, (ii) if $S, T \in \mathcal{W}$, then $S \cap T \in \mathcal{W}$, and (iii) $S \in \mathcal{W}$ or $N \backslash S \in \mathcal{W}$. We have the following familiar property.

Lemma 2.2 Let $\mathcal{W}$ be an ultrafilter on $N$. Then there exists a unique $d \in N$ such that $\{d\} \in \mathcal{W}$.

Proof. By properties (i) and (ii) of an ultrafilter there can be at most one such d. If $N \backslash\{i\} \in \mathcal{W}$ for all $i \in N$ then $\cap_{i \in N} N \backslash\{i\} \in \mathcal{W}$ by (ii), but this violates (i) since $\cap_{i \in N} N \backslash\{i\}=\emptyset$. Hence, there must be a $d \in N$ with $N \backslash\{d\} \notin \mathcal{W}$, so by (iii) we have $\{d\} \in \mathcal{W}$. 


\section{Dictatorship}

Throughout this section, the set $A$ is a polytope with non-empty interior, i.e., the convex hull of a finite set $C=\left\{c_{1}, c_{2}, \ldots, c_{k}\right\} \subseteq \mathbb{R}^{2}$ with $k \geq 3$ where all alternatives in $C$ are extreme points of $A$. Further, $\varphi: A^{N} \rightarrow A$ is a strategyproof and Pareto optimal rule.

We start by showing that only extreme points can be boundary outcomes of $\varphi$.

Lemma 3.1 Let $p \in A^{N}$ and $\varphi(p) \in \partial A$. Then $\varphi(p) \in C$.

Proof. Let $\varphi(p)=c$. To the contrary suppose that $c \notin C$. Then $c \in] \widetilde{c}_{L}, \widetilde{c}_{R}[\subseteq$ $\partial A$ for some $\widetilde{c}_{L}, \widetilde{c}_{R} \in C$. Let $\ell$ be the line through $c$ perpendicular to $] \widetilde{c}_{L}, \widetilde{c}_{R}[$. Line $\ell$ divides $A$ in a left part and a right part, which we denote by $\mathcal{L}$ and $\mathcal{R}$ respectively, more precisely, $\mathcal{L}=H^{\circ}\left(\ell, \widetilde{c}_{L}\right) \cap A$ and $\mathcal{R}=H^{\circ}\left(\ell, \widetilde{c}_{R}\right) \cap A$. For $x$ in $\partial A \cap \mathcal{L}$ consider the perpendicular bisector $\ell_{x, c}$ of $[x, c]$. If $x \notin\left[\widetilde{c}_{L}, c\right]$, let $d_{x}$ be the point of intersection of $\ell_{x, c}$ and $\partial A$ which is not in $H^{\circ}\left(\ell(x, c), \widetilde{c}_{L}\right)$; if $x \in\left[\widetilde{c}_{L}, c\right]$, let $d_{x}$ be the point of intersection of $\ell_{x, c}$ and $\partial A$ which is not in $\left[\widetilde{c}_{L}, c\right]$.

Consider the set $Z$ of points $z \in C \cap \mathcal{L}$ such that $d_{z}$ is in between $d_{z^{\prime}}$ and $c$ for all $z^{\prime} \in C \cap \mathcal{L}$ on the path along the boundary of $A$ passing $\widetilde{c}_{L}$ before $c$. If $Z$ consists of a unique point then call this point $c_{L}$; otherwise, let $c_{L}$ be the point of $Z$ such that $z$ is in between $c_{L}$ and $c$ for all other points $z \in Z{ }^{1}$ We write $d_{L}$ instead of $d_{c_{L}}$. Clearly, $d_{L} \in \partial A \cap \mathcal{L}$ since $d_{\widetilde{c}_{L}} \in \partial A \cap \mathcal{L}$. Now the choice of $c_{L}$ implies that $d_{L} \in H\left(\ell_{z, c}, z\right)$ for all $z \in C \cap \mathcal{L}$, hence that $\left\|d_{L}-z\right\| \leq\left\|d_{L}-c\right\|$ for all $z \in C \cap \mathcal{L}$. In turn, this implies $\left\|d_{L}-x\right\| \leq\left\|d_{L}-c\right\|$ for all $x \in \overline{\mathcal{L}}$.

Let $S_{L}=\left\{i \in N: p(i) \in H^{\circ}\left(\ell_{c_{L}, c}, c_{L}\right)\right\}$. Since $\left\|d_{L}-\widetilde{c}_{L}\right\| \leq\left\|d_{L}-c\right\|$ and $\ell_{\widetilde{c}_{L}, c} \cap A \subseteq \mathcal{L}$ it follows that $\ell_{c_{L}, c} \cap A \subseteq \mathcal{L}$. Pareto optimality of $\varphi$ implies that either $S_{L}$ is non-empty or $p(i) \in \ell_{c_{L}, c} \cap A$ for all $i \in N$ (since otherwise $c_{L}$ would Pareto dominate $c=\varphi(p))$. Now similarly we can define $c_{R}, d_{R}$ and $S_{R}$ for the right part of $A$ and have by Pareto optimality that either $S_{R}$ is non-empty or $p(i) \in \ell_{c_{R}, c} \cap A$ for all $i \in N$. Since $\mathcal{L} \cap \mathcal{R}=\emptyset$ we have that $\ell_{c_{L}, c} \cap A$ and $\ell_{c_{R}, c} \cap A$ are disjoint. This implies that both $S_{L}$ and $S_{R}$ are non-empty.

Now consider the profile $q_{L}$ with $q_{L}(i)=p(i)$ for all $i \notin S_{L}$ and $q_{L}(i)=d_{L}$ for all $i \in S_{L}$. With the aid of the following claim we will prove that $\varphi\left(q_{L}\right)=c_{L}$.

Claim Let $v, w \in A^{N}, i \in S_{L}$, and $a \in H^{\circ}\left(\ell_{c_{L}, c}, c_{L}\right)$ with $v(i)=a, w(i)=d_{L}$, $v(j)=w(j)$ for all $j \in N \backslash\{i\}$, and $\varphi(v) \in\left[d_{L} ; c_{L} \frown c\right]$. Then $\varphi(w) \in\left[d_{L} ; c_{L} \frown c\right]$.

To prove this, first note that $\|a-x\| \leq\|a-c\|$ for all $x \in\left[d_{L} ; c_{L} \frown c\right]$; this is so since $a \in H\left(\ell\left(c, d_{L}\right), c_{L}\right)$ because $c, d_{L} \in \partial A$, and therefore $a \in H\left(\ell_{x, c}, x\right)$ for all $x \in\left[d_{L} ; c_{L} \frown c\right]$. (See Fig. 1(a).) Hence SP of $\varphi$ implies $\|a-c\| \geq\|a-\varphi(w)\|$ otherwise agent $i$ could manipulate from $v$ to $w$. Again by strategy-proofness we have $\left\|d_{L}-\varphi(w)\right\| \geq\left\|d_{L}-c\right\|$ otherwise agent $i$ could manipulate from $w$ to $v$. Suppose that $\left\|\bar{d}_{L}-\varphi(w)\right\|>\left\|d_{L}-c\right\|$. Then, since $c_{L} \in Z$, we have

\footnotetext{
${ }^{1}$ For a polytope of which the boundary is a regular polygon one can show that $c_{L}=\widetilde{c}_{L}$.
} 


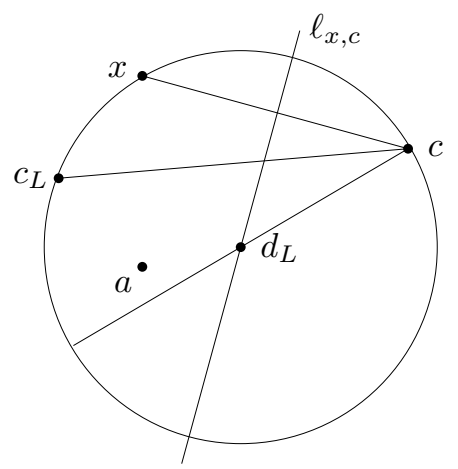

(a)

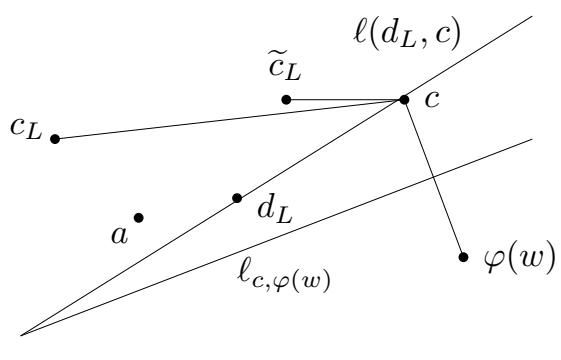

(b)

Figure 1: Illustrating the proof of Lemma 3.1

$\varphi(w) \in \mathcal{R}$. Since $d_{L}$ and $c$ are both in $H\left(\ell_{c, \varphi(w)}, c\right)$ we have $A \cap H\left(\ell\left(d_{L}, c\right), \widetilde{c}_{L}\right) \subseteq$ $H\left(\ell_{c, \varphi(w)}, c\right)$. Since $a \in A \cap H^{\circ}\left(\ell\left(d_{L}, c\right), \widetilde{c}_{L}\right)$ it follows that $a \in H^{\circ}\left(\ell_{c, \varphi(w)}, c\right)$, contradicting $\|a-c\| \geq\|a-\varphi(w)\|$. (See Fig. 1(b).) Therefore we must have $\left\|d_{L}-\varphi(w)\right\|=\left\|d_{L}-c\right\|$.

Now the two circles $\odot\left(d_{L},\left\|d_{L}-c\right\|\right)$ and $\odot(a,\|a-c\|)$ intersect in $c$ and say $c^{\prime}$. Since $\|a-c\| \geq\|a-\varphi(w)\|$ and $\left\|d_{L}-\varphi(w)\right\|=\left\|d_{L}-c\right\|$ it follows that $\varphi(w) \in\left[d_{L} ; c^{\prime} \frown c\right]$. But by the choice of $c_{L}$ we have $\left[d_{L} ; c^{\prime} \frown c\right] \cap A=\left[d_{L} ; c_{L} \frown c\right] \cap A$. Therefore $\varphi(w) \in\left[d_{L} ; c_{L} \frown c\right]$, which completes the proof of the Claim.

Repeated application of the Claim yields that $\varphi\left(q_{L}\right) \in\left[d_{L} ; c_{L} \frown c\right]$. For all $x \in\left[d_{L} ; c_{L} \frown c\right]$ we have by construction that $H\left(\ell_{c_{L}, x}, c_{L}\right) \cap A$ is a subset of $H\left(\ell_{c_{L}, c}, c_{L}\right) \cap A$ and therefore contains no dips of the profile $q_{L}$ other than $d_{L}$. Since $S_{R} \neq \emptyset$, it follows that for profile $q_{L}$ the point $c_{L}$ Pareto dominates dominates all $x \in\left[d_{L} ; c_{L} \frown c\right]$ with $x \neq c_{L}$. Hence, $\varphi\left(q_{L}\right)=c_{L}$.

Next consider the profile $r$ with $r(i)=d_{R}$ for all $i \in S_{R}$ and $r(i)=q_{L}(i)$ for all $i \notin S_{R}$. SP of $\varphi$ implies that $\left\|d_{R}-\varphi(r)\right\| \geq\left\|d_{R}-c_{L}\right\|$. But clearly $d_{R} \in$ $H^{\circ}\left(\ell_{c_{L}, c}, c\right)$ and therefore $\left\|d_{R}-c\right\|<\left\|d_{R}-c_{L}\right\|$. Hence, $\left\|d_{R}-\varphi(r)\right\|>\left\|d_{R}-c\right\|$ and by the the definition of $d_{R}$ (similar to $d_{L}$ above) $\left\|d_{R}-c\right\| \geq\left\|d_{R}-x\right\|$ for all $x \in \overline{\mathcal{R}}$. This implies $\varphi(r) \in \mathcal{L}$. By a completely analogous argument, now starting on the right part of $A$ with a profile $q_{R}$, we can show that $\varphi(r) \in \mathcal{R}$. Since $\mathcal{L} \cap \mathcal{R}=\emptyset$, we have a contradiction, which completes the proof of the lemma.

The next lemma follows by combining Lemmas 2.1 and 3.1 .

Lemma 3.2 Let $a, b \in A$ and $S \subseteq N$. Then $\varphi\left(a^{S}, b^{N \backslash S}\right) \in C$.

For a subset $B$ of $A$ let $A_{B}$ denote the set of alternatives for which $B$ is exactly the set of best alternatives, i.e.,

$$
A_{B}=\{x \in A: \mathfrak{b}(x)=B\} .
$$


For a singleton $\{a\}$ we write $A_{a}$ instead of $A_{\{a\}}$. We can make the following observations. If $B \subseteq A$ and $B \nsubseteq C$, then $A_{B}=\emptyset$. If $B=\{a, b\}$ for some distinct $a, b \in C$ then $A_{B} \subseteq \ell_{a, b}$ and if $B=\{a, b, c\}$ for some distinct $a, b, c \in C$ then $A_{B} \subseteq \ell_{a, b} \cap \ell_{b, c}$. Moreover, if $A_{a} \neq \emptyset$ for some $a \in C$ then $A_{a}$ is convex. This can be seen as follows. Let $x, y \in A_{a}$ and let $b \in A \backslash\{a\}$, then $x, y \in H^{\circ}\left(\ell_{a, b}, b\right)$, hence $z \in H^{\circ}\left(\ell_{a, b}, b\right)$ for any convex combination $z$ of $x$ and $y$, so $b \notin \mathfrak{b}(z)$. Since $b$ was arbitrary and $\mathfrak{b}(z) \neq \emptyset$, this implies that $z \in A_{a}$. Let

$$
\mathcal{B}=\left\{a \in A: A_{a} \neq \emptyset\right\} .
$$

Elements of $\mathcal{B}$ are called single-best alternatives. Combining our observations we see that for each $a \in \mathcal{B}$ the set $A_{a}$ is a convex polygon which is open relative to $A$, and that $A=\cup_{x \in \mathcal{B}} \bar{A}_{x}$.

The following two lemmas establish further facts about alternatives in $\mathcal{B}$.

Lemma 3.3 Let $a, b$, and $c$ be three distinct alternatives in $\mathcal{B}$. Then $m_{a, b, c}$ exists and $m_{a, b, c} \in A^{\circ}$.

Proof. Since $a, b, c \in C$ they are not collinear and thus $m_{a, b, c}$ exists. The straight lines $\ell_{a, b}, \ell_{a, c}$, and $\ell_{b, c}$ intersect at $m_{a, b, c}$ and determine six open nonempty regions which divide $A^{\circ} \backslash\left(\ell_{a, b} \cup \ell_{a, c} \cup \ell_{b, c}\right)$ into disjoint sets. A single dipped preference $R_{x}$ is constant over $a, b$, and $c$ as long as $x$ stays in one of these regions. Call these regions $V_{a b c}, V_{b a c}, V_{b c a}, V_{c b a}, V_{c a b}$ and $V_{a c b}$; for $x \in V_{a b c}$ we have that $a$ is (strictly) preferred over $b$ and $b$ over $c$ according to $R_{x}$, and similarly for the other five regions. Now $A_{a} \subseteq\left(V_{a b c} \cup V_{a c b} \cup \ell_{b, c}\right)^{\circ} \cap A$. Since $A_{a}$ is non-empty we have that $\left(V_{a b c} \cup V_{a c b} \cup \ell_{b, c}\right)^{\circ} \cap A$ is non-empty and therefore $\left(V_{a b c} \cup V_{a c b} \cup \ell_{b, c}\right)^{\circ} \cap A^{\circ}$ is non-empty. Similarly $\left(V_{b c a} \cup V_{b a c} \cup \ell_{a, c}\right)^{\circ} \cap A^{\circ}$ and $\left(V_{c a b} \cup V_{c b a} \cup \ell_{a, b}\right)^{\circ} \cap A^{\circ}$ are non-empty. Take points $x_{a}, x_{b}, x_{c}$ in these respective regions, then $m_{a, b, c}$ is in the convex hull of these three points and is therefore an interior point of $A$.

Lemma 3.4 Let $a \in \mathcal{B}$. Then $A^{\circ} \backslash \bar{A}_{a}$ is connected.

Proof. If $A^{\circ} \backslash \bar{A}_{a}=A_{b}$ for some $b \in \mathcal{B}$ we are done because $A_{b}$ is obviously connected as it is convex. Therefore let $y, z \in A^{\circ}$ with $y \in A_{b}$ and $z \in A_{c}$ for some distinct $b, c \in \mathcal{B} \backslash\{a\}$. Since $A^{\circ} \subseteq \cup\left\{\bar{A}_{x}: x \in \mathcal{B}\right\}$ it is sufficient to prove that there is a path in $A^{\circ} \backslash \bar{A}_{a}$ connecting $y$ and $z .^{2}$ Lemma 3.3 implies that $m_{a, b, c} \in A^{\circ}$. Convexity of $A$ implies that $A^{\circ}$ is convex. Hence, $\left[y, m_{a, b, c}\right] \in A^{\circ}$ and $\left[z, m_{a, b, c}\right] \in A^{\circ}$. With notations as in the proof of Lemma 3.3 we may further conclude that $\left[y, m_{a, b, c}\left[\subseteq\left(V_{b c a} \cup V_{b a c} \cup \ell_{a, c}\right)^{\circ}\right.\right.$ and $\left[z, m_{a, b, c}\left[\subseteq\left(V_{c a b} \cup V_{c b a} \cup \ell_{a, b}\right)^{\circ}\right.\right.$. Since $A_{a} \subseteq\left(V_{a b c} \cup V_{a c b} \cup \ell_{b, c}\right)^{\circ} \cap A$ and $\left(V_{a b c} \cup V_{a c b} \cup \ell_{b, c}\right)^{\circ}$ is disjoint from both $\left(V_{b c a} \cup V_{b a c} \cup \ell_{a, c}\right)^{\circ}$ and $\left(V_{c a b} \cup V_{c b a} \cup \ell_{a, b}\right)^{\circ}$ it follows that we can construct a path from $y$ to $z$ in $A^{\circ} \backslash \bar{A}_{a}$, as follows. Choose points $y^{\prime} \in\left[y, m_{a, b, c}[\right.$ and $\left.\left.z^{\prime} \in\right] m_{a, b, c}, z\right]$ close enough to $m_{a, b, c}$ such that there is a path $P$ from $y^{\prime}$ to $z^{\prime}$ in $A^{\circ}$ disjoint from $\bar{A}_{a}$; then the desired path is $\left[y, y^{\prime}\right] \cup P \cup\left[z^{\prime}, z\right]$. (See Fig. 2.)

\footnotetext{
${ }^{2}$ I.e. the graph of a continuous function $[0,1] \rightarrow A$ with $0 \mapsto y$ and $1 \mapsto z$.
} 


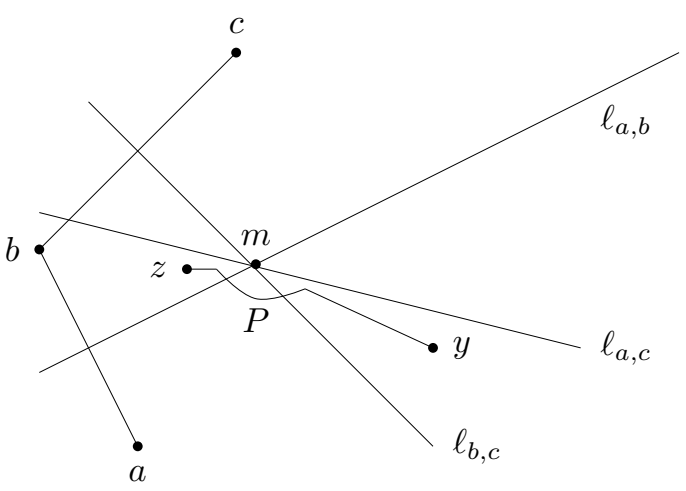

Figure 2: Illustrating the proof of Lemma 3.4: path between $y$ and $z ; m=m_{a, b, c}$

In what follows we often use a notation like $R=\ldots x \ldots y z \ldots(x, y, z \in C)$ to express that, in the preference $R, x$ is strictly preferred to $y$, and $y$ to $z$ such that other alternatives in $C$ are either above $y$ or below $z$ in preference. The following lemma is a simple consequence of (intermediate) strategy-proofness. We leave its proof to the reader.

Lemma 3.5 Let $\emptyset \neq S \subseteq N, p, q \in A^{N}$ and $x, y \in C$ with $x \neq y$ such that ( $i$ ) $p(j)=q(j)$ for all $j \in N \backslash S ;(i i) p(i)=p(j)$ and $q(i)=q(j)$ for all $i, j \in S$; (iii) for all $i \in S, p(i)$ and $q(i)$ order the alternatives of $C$ strictly and coincide on $C$ except for a swap between $x$ and $y: p(i)=\ldots x y \ldots, q(i)=\ldots y x \ldots ;$ and $(i v)$ $\varphi(p), \varphi(q) \in C, \varphi(p) \neq \varphi(q)$. Then $x=\varphi(p)$ and $y=\varphi(q)$.

Lemma 3.5 says that if an agent's preference changes by swapping two alternatives in $C$ while not changing the (strict) order between other alternatives in $C$, and if therefore the alternative assigned by the rule changes, then the two swapped alternatives must be the alternatives assigned by the rule. ${ }^{3}$

The next result can be interpreted as saying that $\varphi$ leaves no room for compromising at two-dip profiles.

Lemma 3.6 Let $S \subseteq N, a, b \in \mathcal{B}, x \in A_{a}, y \in A_{b}$, and $p=\left(x^{S}, y^{N \backslash S}\right) \in A^{N}$. Then $\varphi(p) \in\{a, b\}$.

Proof. If $a=b$ then the claim in the lemma follows from Pareto-optimality. Thus, let $a \neq b$ and suppose that $\varphi(p)=c \notin\{a, b\}$. We derive a contradiction. Pareto optimality implies $R_{x}=a \ldots c \ldots b \ldots$ and $R_{y}=b \ldots c \ldots a \ldots$ We take a path from $x$ to $c$ with the properties that (i) along this path, $c$ can only become worse compared to other elements of $C$; (ii) along this path the preference over elements of $C$ changes by swaps of at most two alternatives at the same time; and (iii) along this path preferences over $C$ are strict except at swaps as in (ii). Formally, this path is the graph of a continuous function $\pi$ from $[0,1]$ to $A$ with

\footnotetext{
${ }^{3}$ This is a restricted version of (Maskin) monotonicity, which is implied by strategyproofness.
} 
$\pi(0)=x$ and $\pi(1)=c$ such that (i) for all $t_{1}<t_{2}$ and $z \in C$, if $(z, c) \in R_{\pi\left(t_{1}\right)}$ then $(z, c) \in R_{\pi\left(t_{2}\right)}$ and $(c, z) \notin R_{\pi\left(t_{2}\right)}$; (ii) for all $t \in[0,1]$ there are no distinct $z, z^{\prime}, z^{\prime \prime} \in C$ such that $\pi(t) \in \ell_{z, z^{\prime}} \cap \ell_{z, z^{\prime \prime}}$; and (iii) there are (only) finitely many $t$ for which $\pi(t) \in \ell_{z, z^{\prime}}$ for some $z, z^{\prime} \in C$. We call a pair $R_{\pi\left(t_{1}\right)}, R_{\pi\left(t_{2}\right)}$ an 'elementary change' if the latter preference arises from the former after swapping two adjacent alternatives in $C$.

Let $p^{t}=\left(\pi(t)^{S}, y^{N \backslash S}\right)$. Then $\varphi\left(p^{1}\right) \neq c$ since $c$ is not Pareto optimal at $p^{1}$. Thus, there must be a first elementary change such that $\varphi\left(p^{t_{1}}\right)=c$ and $\varphi\left(p^{t_{2}}\right)=d \neq c$. By Lemma 3.5 this implies that $R_{\pi\left(t_{1}\right)}=\ldots c d \ldots$ whereas $R_{\pi\left(t_{2}\right)}=\ldots d c \ldots$

Now there are two cases.

Case $1 R_{y}=b \ldots d \ldots c \ldots a \ldots$

Then in the same way as above we can find a path $\rho$ from $y$ to $c$, profiles $q^{t}=\left(\pi\left(t_{1}\right)^{S}, \rho(t)^{N \backslash S}\right)$ and $t_{3}$ and $t_{4}$ such that $\varphi\left(q^{t_{3}}\right)=c, \varphi\left(q^{t_{4}}\right)=e \neq c$ and $R_{\rho\left(t_{3}\right)}$ and $R_{\rho\left(t_{4}\right)}$ form an elementary change swapping $c$ and $e$. Consider $r=\left(\pi\left(t_{2}\right)^{S}, \rho\left(t_{4}\right)^{N \backslash S}\right)$ and $u^{t}=\left(\pi(t)^{S}, \rho\left(t_{3}\right)^{N \backslash S}\right)$ for $t \in\left[t_{1}, t_{2}\right]$. So, $\varphi\left(u^{t_{1}}\right)=$ $\varphi\left(q^{t_{3}}\right)=c$. Let $\varphi(r)=f$. Note that $R_{\rho\left(t_{3}\right)}=\ldots d \ldots c e \ldots$ and $R_{\rho\left(t_{4}\right)}=\ldots d \ldots e c \ldots$. Since $\varphi\left(u^{t_{1}}\right)=c$ it follows by Pareto optimality and Lemma 3.5 that $\varphi\left(u^{t_{2}}\right)=d$. Summarizing we have:

\begin{tabular}{|c|c|c|}
\hline \hline & $u^{t_{1}}=q^{t_{3}}$ & $q^{t_{4}}$ \\
\hline$i \in S$ & $u^{t_{1}}(i)=R_{\pi\left(t_{1}\right)}=\ldots c d \ldots$ & $q^{t_{4}}(i)=R_{\pi\left(t_{1}\right)}=\ldots c d \ldots$ \\
$i \in N \backslash S$ & $u^{t_{1}}(i)=R_{\rho\left(t_{3}\right)}=\ldots d \ldots c e \ldots$ & $q^{t_{4}}(i)=R_{\rho\left(t_{4}\right)}=\ldots d \ldots e c \ldots$ \\
\hline & $\varphi\left(u^{t_{1}}\right)=c$ & $\varphi\left(q^{t_{4}}\right)=e$ \\
\hline \hline & $u^{t_{2}}$ & $r$ \\
\hline$i \in S$ & $u^{t_{2}}(i)=R_{\pi\left(t_{2}\right)}=\ldots d c \ldots$ & $r(i)=R_{\pi\left(t_{2}\right)}=\ldots d c \ldots$ \\
$i \in N \backslash S$ & $u^{t_{2}}(i)=R_{\rho\left(t_{3}\right)}=\ldots d \ldots c e \ldots$ & $r(i)=R_{\rho\left(t_{4}\right)}=\ldots d \ldots e c \ldots$ \\
\hline & $\varphi\left(u^{t_{2}}\right)=d$ & $\varphi(r)=f$ \\
\hline \hline
\end{tabular}

There are two subcases.

Subcase $d \neq f$. Comparing $u^{t_{2}}$ and $r$, Lemma 3.5 implies $R_{\rho\left(t_{3}\right)}=\ldots d f \ldots c e \ldots$ and $R_{\rho\left(t_{4}\right)}=\ldots f d \ldots e c \ldots$ So $c, d, e$, and $f$ are all different. Comparing $q^{t_{4}}$ and $r$, Lemma 3.5 implies (i) $R_{\pi\left(t_{1}\right)}=\ldots e f \ldots c d \ldots$ and $R_{\pi\left(t_{2}\right)}=\ldots f e \ldots d c \ldots$ or (ii) $R_{\pi\left(t_{1}\right)}=\ldots c d \ldots e f \ldots$ and $R_{\pi\left(t_{2}\right)}=\ldots d c \ldots f e \ldots$ But (i) implies that $\varphi\left(u^{t_{1}}\right)=c$ is Pareto dominated by $f$ at $u^{t_{1}}$, a contradiction; and (ii) implies that $\varphi\left(q^{t_{4}}\right)=e$ is Pareto dominated by $d$ at $q^{t_{4}}$, likewise a contradiction. So this subcase cannot occur.

Subcase $d=f$. Comparing $q^{t_{4}}$ and $r$, Lemma 3.5 implies $R_{\pi\left(t_{1}\right)}=\ldots e d \ldots$ and $R_{\pi\left(t_{2}\right)}=\ldots d e \ldots$ This implies the contradiction $c=e$ and therefore also this subcase cannot occur. This ends the proof of Case 1.

Case $2 R_{y}=b \ldots c \ldots d \ldots a \ldots$

By interchanging the roles of $c$ and $d$ and thus those of $R_{\pi\left(t_{1}\right)}$ and $R_{\pi\left(t_{2}\right)}$, and then proceeding like in Case 1 , we derive a similar contradiction. 
Call a coalition $S \subseteq N$ quasi-decisive (given $\varphi$ ) if $\varphi(p)=a$ for all $a, b \in A$ and every profile $p=\left(x^{S}, y^{N \backslash S}\right)$ with $x \in A_{a}$ and $y \in A_{b}$.

Lemma 3.7 Let $|\mathcal{B}| \geq 3$. Then for every $S \subseteq N$, either $S$ or $N \backslash S$ is quasidecisive.

Proof. Let $S \subseteq N$. If $S=N$ or $S=\emptyset$ then we are done by Pareto optimality. So assume $S \neq \emptyset, N$. Clearly, $S$ and $N \backslash S$ cannot both be quasi-decisive. Let $a, b$ be two different elements of $\mathcal{B}$, and let $x \in A_{a}, y \in A_{b}$, and $p=\left(x^{S}, y^{N \backslash S}\right)$. By Lemma 3.6, $\varphi(p) \in\{a, b\}$. Without loss of generality assume that $\varphi(p)=a$. Take $c, d \in \mathcal{B}, v \in A_{c}$ and $w \in A_{d}$ arbitrary, and let $q=\left(v^{S}, w^{N \backslash S}\right)$. It is sufficient to prove that $\varphi(q)=c$. By Pareto optimality we may assume that $c \neq d$. Lemma 3.6 implies $\varphi(q) \in\{c, d\}$. We proceed in four steps.

Step 1 Assume $v=x$.

Then, since $v=x$ we have $c=a$. If also $b=d$, then $\varphi(q)=c=a$ otherwise coalition $N \backslash S$ could manipulate from $p$ to $q$ and obtain $d=b$. So assume $b \neq d$. By Lemma 3.4 there is a path from $y$ to $w$ disjoint from $\bar{A}_{a}$. Along this path we can find points $y=u_{1}, u_{2}, \ldots, u_{k}=w(k \geq 2)$ such that $u_{t} \in A_{f_{t}}, f_{t} \in \mathcal{B}$, for $t=1, \ldots, k$ (so $f_{1}=b, f_{k}=d$ ) and such that for every $t=1, \ldots, k-1$ we have $R_{u_{t \mid \mathcal{B}}}=f_{t} f_{t+1} \ldots$ and $R_{u_{t+1} \mid \mathcal{B}}=f_{t+1} f_{t} \ldots{ }^{4}$ For every $t=1, \ldots, k$ let $p^{t}=\left(x^{S}, u_{t}^{N \backslash S}\right)$. Thus, $p^{1}=p$ and $p^{k}=q$. Since $\varphi\left(p^{1}\right)=a, f_{1}, f_{2} \neq a, R_{u_{2} \mid \mathcal{B}}=f_{2} f_{1} \ldots$, and $\varphi\left(p^{2}\right) \in\left\{a, f_{2}\right\}$ by Lemma 3.6, we have by strategy-proofness that $\varphi\left(p^{2}\right)=a$, since otherwise coalition $N \backslash S$ could manipulate from $p^{1}=p$ to $p^{2}$, obtaining $f_{2}$ instead of $a$. Similarly, it follows from this that $\varphi\left(p^{3}\right)=a$ and so on, so that $a=\varphi\left(p^{k}\right)=\varphi(q)$.

Step 2 Assume $v \neq x$ and $d \neq a$.

Consider $p^{\prime}=\left(x^{S}, w^{N \backslash S}\right)$. Then Step 1 implies $\varphi\left(p^{\prime}\right)=a$. By Step 1 applied to $p^{\prime}$ and $q$, if $\varphi(q)=d$ then $\varphi\left(p^{\prime}\right)=d$, a contradiction since $d \neq a$. Hence $\varphi(q)=c$.

Step 3 Assume $y=v, w=x$.

Let $e \in \mathcal{B} \backslash\{a, b\}$ and $u \in A_{e}$. Then $\varphi(p)=\varphi\left(x^{S}, y^{N \backslash S}\right)=a$ implies by Step 1 that $\varphi\left(x^{S}, u^{N \backslash S}\right)=a$. In turn, by Step 2 this implies that $\varphi\left(y^{S}, u^{N \backslash S}\right)=b$, which by Step 1 again yields $\varphi(q)=\varphi\left(y^{S}, x^{N \backslash S}\right)=b$. Since $y=v$, we have $b=c$, so $\varphi(q)=c$.

Step 4 Assume $v \neq x, d=a$.

Then $\varphi(p)=\varphi\left(x^{S}, y^{N \backslash S}\right)=a$ implies by Step 3 that $\varphi\left(y^{S}, x^{N \backslash S}\right)=b$, hence by Step 1 we obtain $\varphi\left(y^{S}, w^{N \backslash S}\right)=b$. If $y=v$, hence $b=c$, we have $\varphi(q)=\varphi\left(v^{S}, w^{N \backslash S}\right)=\varphi\left(y^{S}, w^{N \backslash S}\right)=b=c$. If $y \neq v$ then $d=a \neq b$ and Step 2 imply $\varphi(q)=\varphi\left(v^{S}, w^{N \backslash S}\right)=c$.

Since the case $v=x$ is covered in Step 1, the case $v \neq x$ and $d \neq a$ in Step 2, and the case $v \neq x$ and $d=a$ in Step 4, all cases are covered, so the proof is complete.

\footnotetext{
${ }^{4} R_{x \mid D}$ denotes the restriction of preference $R_{x}$ to $D \subseteq A$.
} 
Lemma 3.8 Let $|\mathcal{B}| \geq 3$. Then for every $S \subseteq N$, either $S$ or $N \backslash S$ is decisive.

Proof. Let $S \subseteq N$. Clearly, $S$ and $N \backslash S$ cannot both be decisive. By Lemma 3.7 either $S$ or $N \backslash S$ is quasi-decisive. Assume that $S$ is quasi-decisive, the other case is analogous. Consider a profile $p$ with $p(i)=x$ for all $i \in S$ such that $x \in A_{a}$ for some $a \in \mathcal{B}$. We first prove that $\varphi(p)=a$. Take a $d \in \mathcal{B}$ such that $a \in \partial A_{d}$, and take a $v \in A_{d}$. Let $r=\left(x^{S}, v^{N \backslash S}\right)$. Quasi-decisiveness of $S$ implies that $\varphi(r)=a$. By applying intermediate strategy-proofness to the profiles $r$ and $p$ we obtain $\|v-a\| \geq\|v-\varphi(p)\|$. Since we can take $v$ as close to $a$ as desired, this implies $\varphi(p)=a$. Finally, let $q$ be a profile with $q(i)=y \in A$ for all $i \in S$, and suppose that $\varphi(q) \notin \mathfrak{b}(y)$. Then take $b \in \mathfrak{b}(y) \cap \mathcal{B}$ (this is possible since $\left.A=\cup\left\{\bar{A}_{x}: x \in \mathcal{B}\right\}\right)$ and $z \in A$ such that $z \in A_{b}$. By the first part of the proof, $\varphi\left(q^{\prime}\right)=b$ where $q^{\prime}(i)=z$ for all $i \in S$ and $q^{\prime}(i)=q(i)$ for all $i \in N \backslash S$. Then $S$ can manipulate from $q$ to $q^{\prime}$, violating (intermediate) SP.

In what follows we pin down those polytopes $A$ for which the intersection of two decisive sets is again decisive. Call a finite subset of $\mathbb{R}^{2}$ rectangular if it consists of exactly four points which are the vertices of one and the same rectangle.

Lemma 3.9 Let the following conditions hold:

(i) $|\mathcal{B}| \geq 3$.

(ii) There are distinct $a, b, c \in \mathcal{B}$ such that $m_{a, b, c} \in \partial A_{a} \cap A^{\circ}$ and such that there is no $x \in \mathcal{B}$ for which $\{a, b, c, x\}$ is rectangular.

Let $S, T \subseteq N$ be both decisive. Then $S \cap T$ is decisive.

Proof. Suppose, to the contrary (cf. Lemma 3.8), that $N \backslash(S \cap T)$ is decisive. Then we have a partition $X=S \cap T, Y=S \backslash T$ and $Z=N \backslash S$ of $N$ such that $X, Y$ and $Z$ are not decisive. Hence any union of two of these sets, being the complement of the third one, is decisive. We will derive a contradiction.

Let $a, b$ and $c$ be as in (ii) in the statement of the lemma. We choose $\widetilde{a} \in A^{\circ}$ close to $a$ and $\bar{b}, \bar{c}, \bar{a}_{b}, \bar{a}_{c} \in A^{\circ}$ close to $m:=m_{a, b, c}$ (how close will be specified below) with

$$
\begin{aligned}
R_{\bar{b}} & =b a \ldots c \ldots \\
R_{\bar{c}} & =c a \ldots b \ldots \\
R_{\bar{a}_{b}} & =a b \ldots c \ldots \\
R_{\bar{a}_{c}} & =a c \ldots b \ldots \\
R_{\widetilde{a}} & =\ldots c \ldots b \ldots a
\end{aligned}
$$

and such that all these preferences (restricted to $C$ ) are strict. (This is possible in particular since $m_{a, b, c} \in \partial A_{a} \cap A^{\circ}$. (See Fig. 3(a).) If $\widehat{a}$ cannot be chosen this way then we choose it such that $R_{\widehat{a}}=\ldots b \ldots c \ldots a$ and proceed analogously.) By choosing these points close enough to $a$ and $m$, respectively, we have that for 


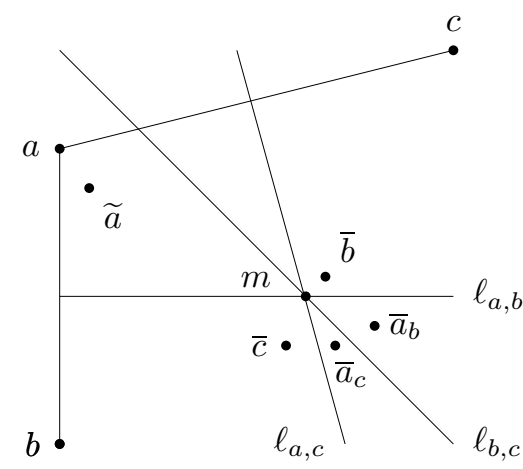

(a)

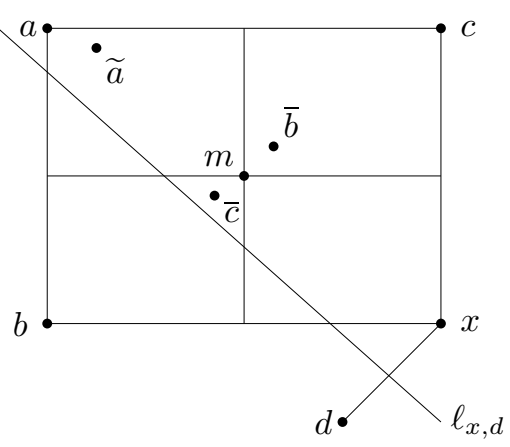

(b)

Figure 3: Illustrating the proof of Lemma 3.9

any profile $q$ with dips at these points, any alternative in $A^{\circ}$ is Pareto dominated by a boundary point. Hence, by Lemma 3.1, $\varphi(q) \in C$ for such profiles $q$. In particular, $\varphi$ assigns an alternative in $C$ to any of the following profiles:

$$
\begin{aligned}
p & =\left(\widetilde{a}^{X}, \bar{b}^{Y}, \bar{c}^{Z}\right) \\
p^{\bar{a}_{b}} & =\left(\widetilde{a}^{X}, \bar{a}_{b}^{Y}, \bar{c}^{Z}\right) \\
p^{\bar{a}_{c}} & =\left(\widetilde{a}^{X}, \bar{b}^{Y}, \bar{a}_{c}^{Z}\right) \\
r & =\left(\widetilde{a}^{X}, \bar{a}_{b}^{Y}, \bar{a}_{c}^{Z}\right) .
\end{aligned}
$$

Claim $\varphi(p) \in\{a, b, c\}$.

Let $\varphi(p)=x, \varphi\left(p^{\bar{a}_{b}}\right)=y$ and $\varphi\left(p^{\bar{a}_{c}}\right)=z$. Since $Y \cup Z$ is decisive and since $\bar{a}_{b}, \bar{a}_{c} \in A_{a}$, we have by strategy-proofness that $\varphi(r)=a$.

First consider $r$ and $p^{\bar{a}_{b}}$. By Lemma 3.5 it follows that the first change, starting from below, in the preferences $R_{\bar{a}_{c}}$ and $R_{\bar{c}}$ must be a swap between $\varphi(r)$ and $\varphi\left(p^{\bar{a}_{b}}\right)$. Since $\varphi(r)=a$, this implies that either (i.a) $y=a$ or (i.b) $y=c$.

Similarly, comparing $r$ and $p^{\bar{a}_{c}}$ yields either (ii.a) $z=a$ or (ii.b) $z=b$.

We next compare $p$ and $p^{\bar{a}_{b}}$. These profiles differ for the agents in $Y$, who have dips $\bar{b}$ in $p$ and dips $\bar{a}_{b}$ in $p^{\bar{a}_{b}}$. By going from $\bar{b}$ to $\bar{a}_{b}$ only the perpendicular bisector $\ell_{a, b}$ is crossed. By Lemma 3.5, as before the first change in preference from below must be a swap between $\varphi(p)=x$ and $\varphi\left(p^{\bar{a}_{b}}\right)=y$, but this implies that $\ell_{x, y}$ coincides with $\ell_{a, b}$. Therefore, we now have either (iii.a) $x=y$ or (iii.b) $x \neq y$ and $\sigma_{a, b}(x)=y$.

Similarly, comparing $p$ and $p^{\bar{a}_{c}}$ yields either (iv.a) $x=z$ or (iv.b) $x \neq z$ and $\sigma_{a, c}(x)=z$.

If $x=y$ or $x=z$, then (i) or (ii) implies that $x \in\{a, b, c\}$. Let $x \neq y, x \neq z$. Then $\sigma_{a, b}(x)=y$ and $\sigma_{a, c}(x)=z$ by (iii.b) and (iv.b). Since $a, b, c$ are distinct and thus $\ell_{a, b} \neq \ell_{a, c}$ it follows that $z \neq y$. If $y=a$ or $z=a$, then $\sigma_{a, b}(x)=y$ and $\sigma_{a, c}(x)=z$ imply $x=b$ or $x=c$, hence we are done again. So, assume $y \neq a$ and $z \neq a$, then $y=c$ by (i.b) and $z=b$ by (ii.b). Then by (iii.b) and 
(iv.b) we have $\sigma_{a, b}(x)=y=c$ and $\sigma_{a, c}(x)=z=b$. We now assume $a \neq x$ and derive a contradiction, which will complete the proof of the Claim. Since $\sigma_{a, c}(a)=c$ and $\sigma_{a, b}(a)=b$ the assumption $a \neq x$ implies that $a, b, c$ and $x$ are vertices of a rectangle. (See Fig. 3(b).) To derive the contradiction it is, in view of condition (ii) in the lemma, sufficient to prove that $x \in \mathcal{B}$. Let $d \in \mathfrak{b}\left(R_{a}\right)$, hence $d \notin\{a, b, c\}$, with $d \neq x$. We will show that $m \in \ell_{x, d}$. This implies that $a, x$, and $d$ are on the same circle with center $m$. Since $a$ and $x$ are on the same diameter in this circle we have that $\|d-a\|<\|x-a\|$, contradicting $d \in \mathfrak{b}\left(R_{a}\right)$. So $a \in A_{x}$, hence $x \in \mathcal{B}$.

We are left to show that $m \in \ell_{x, d}$. Suppose, to the contrary, that $m \notin \ell_{x, d}$. Since $d \in \mathfrak{b}\left(R_{a}\right)$ we have $a \in H\left(\ell_{x, d}, x\right)$, hence $m \in H^{\circ}\left(\ell_{x, d}, x\right)$ and therefore $\bar{b}, \bar{c} \in H^{\circ}\left(\ell_{x, d}, x\right)$ by the choice of these points (note that these points can be chosen close enough to $m$ to achieve this, given that there are only finitely many candidates for the points $x$ and $d$ ). By the choice of $\widetilde{a}$, also $\widetilde{a} \in H^{\circ}\left(\ell_{x, d}, x\right)$. But this implies that $d$ Pareto dominates $x$ at profile $p$, contradicting $\varphi(p)=x$. This completes the proof of the Claim.

Consider the profile $p^{\prime}=\left(\bar{c}^{X}, \bar{b}^{Y}, \bar{c}^{Z}\right)$. Since $X \cup Z$ is decisive we have that $\varphi\left(p^{\prime}\right)=c$. Strategy-proofness and the Claim now imply that $x=\varphi(p)=c$.

Next we prove that $\varphi\left(p^{\bar{a}_{c}}\right)=z \in\{a, b\}$. Note that $z \in \mathcal{C}$. Because of $\varphi(r)=a$, strategy-proofness implies that $z$ is weakly preferred to $a$ at $R_{\bar{b}}$. So, $z \in\{a, b\}$.

Let $p^{\prime \prime}=\left(\bar{b}^{X}, \bar{b}^{Y}, \bar{a}_{c}^{Z}\right)$. Since $X \cup Y$ is decisive we have $\varphi\left(p^{\prime \prime}\right)=b$. Then strategy-proofness implies $\varphi\left(p^{\bar{a}_{c}}\right)=z=b$. Since $\varphi(p)=c$, coalition $Z$ can manipulate from $p^{\bar{a}_{c}}$ to $p$, violating strategy-proofness.

Before continuing with the main result of this section a consideration of the polytopes to which the preceding lemmas apply is in order. First, let $|C|=3$, i.e., $A$ is a triangle including its interior. If this triangle is acute then $\mathcal{B}=C$, hence $|\mathcal{B}|=3$ and also condition (ii) of Lemma 3.9 is trivially satisfied. If this triangle is not acute, however, then $|\mathcal{B}|=2$ and this case will be studied in the next section. Second, let $|C|>3$. If $|\mathcal{B}|=2$, which means, roughly, that the polytope $A$ is rather flat, then again the results in the next section apply. For cases where $|\mathcal{B}| \geq 3$ we provide an exact characterization of those polytopes where condition (ii) of Lemma 3.9 is not satisfied. We start with an auxiliary lemma.

Lemma 3.10 Let $|\mathcal{B}| \geq 3$ and let $a \in \mathcal{B}$. Then there are distinct $b, c \in \mathcal{B} \backslash\{a\}$ such that $m_{a, b, c} \in A^{\circ} \cap \partial A_{a}$.

Proof. For any two distinct points $x, y \in \mathcal{B} \backslash\{a\}$, we have $m_{a, x, y} \in A^{\circ}$ by Lemma 3.3. Now, since $\mathcal{B}$ is finite there must be distinct $b, c \in \mathcal{B} \backslash\{a\}$ such that the set $H^{\circ}\left(\ell_{a, b}, b\right) \cap H^{\circ}\left(\ell_{a, c}, b\right)$ contains no point of the form $m_{a, x, y}$ for distinct $x, y \in \mathcal{B} \backslash\{a\}$ other than $m_{a, b, c}$. We claim that $H^{\circ}\left(\ell_{a, b}, b\right) \cap H^{\circ}\left(\ell_{a, c}, c\right) \cap A$ also has empty intersection with $\ell_{a, x}$ for any $x \in C \backslash \mathcal{B}$. If not, then there is such an $x$ and a point $x_{a} \in H^{\circ}\left(\ell_{a, b}, b\right) \cap H^{\circ}\left(\ell_{a, c}, b\right) \cap H^{\circ}\left(\ell_{a, x}, x\right) \cap A$ with $R_{x_{a}}=a x \ldots$ and such that $R_{\sigma_{a, x}\left(x_{a}\right)}=x a \ldots$ But then $\sigma_{a, x}\left(x_{a}\right) \in A_{x}$, so $x \in \mathcal{B}$, a contradiction. 
It follows that $H^{\circ}\left(\ell_{a, b}, b\right) \cap H^{\circ}\left(\ell_{a, c}, b\right) \cap A \subseteq A_{a}$. Since $m_{a, b, c}=\ell_{a, b} \cap \ell_{a, c}$ this implies that $m_{a, b, c} \in \bar{A}_{a}$. Since, clearly, $m_{a, b, c} \notin A_{a}$ we have $m_{a, b, c} \in \partial A_{a}$.

The next lemma characterizes those polytopes where condition (ii) of Lemma 3.9 is not satisfied.

Lemma 3.11 Let $|\mathcal{B}| \geq 3$. Then condition (ii) in Lemma 3.9 does not hold if and only if $\mathcal{B}$ is rectangular.

Proof. For the only-if direction, assume that condition (ii) in Lemma 3.9 does not hold. We show that $\mathcal{B}$ is rectangular. The proof of this fact proceeds in a few steps. We start with stating (without proof) the following useful fact.

Step 1 A set $Q \subseteq \mathbb{R}^{2}$ is an orthant if it is of the form $Q=\left\{p+\lambda_{1} x^{1}+\lambda_{2} x^{2} \mid\right.$ $\left.\lambda_{1}, \lambda_{2} \geq 0\right\}$ for some $p \in \mathbb{R}^{2}$ and some perpendicular vectors $x^{1}, x^{2} \in \mathbb{R}^{2} \backslash\{0\}$. Denote $p=p(Q)$. Let $X$ be a compact and convex subset of $\mathbb{R}^{2}$ with non-empty interior, written as $X=\bigcup_{i \in I}\left(Q^{i} \cap X\right)$ where $I$ is some index set, $Q^{i}$ is an orthant with $p\left(Q^{i}\right) \in X^{\circ}$ for each $i \in I$, and $\left(Q^{j} \cap X\right)^{\circ} \cap\left(Q^{k} \cap X\right)^{\circ}=\emptyset$ for all distinct $j, k \in I$. Then $|I|=4$.

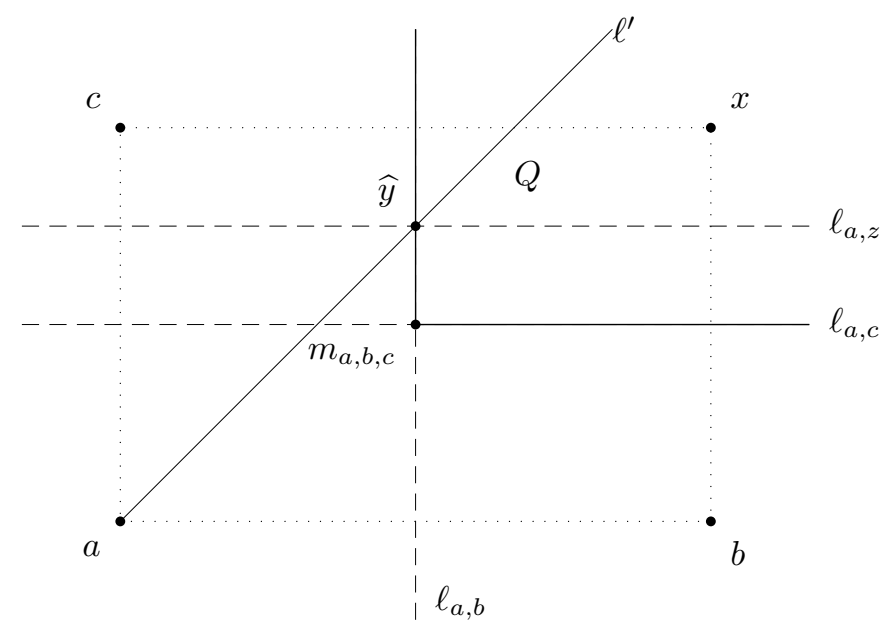

Figure 4: Illustrating the proof of Lemma 3.11, Step 2

Step 2 Let $a \in \mathcal{B}$ and let $b, c \in \mathcal{B} \backslash\{a\}, b \neq c$, such that $m_{a, b, c} \in A^{\circ} \cap \partial A$. Since condition (ii) in Lemma 3.9 does not hold, there is an $x \in \mathcal{B}$ such that $\{a, b, c, x\}$ is rectangular. Without loss of generality assume that $\ell(a, b)$ and $\ell(c, x)$, as well as $\ell(a, c)$ and $\ell(b, x)$ are parallel. (See Fig. 4 for an illustration of the proof of this step.) Denote $Q=H\left(\ell_{a, b}, x\right) \cap H\left(\ell_{a, c}, x\right)$. Then $A_{a}=Q^{\circ} \cap A$.

In order to prove this claim, we first observe the following. Let $y$ be any point of $\bar{A}_{a}$ and let $\ell$ be the line through $a$ and $y$. Then $y^{\prime} \in A_{a}$ for any point $y^{\prime} \in A$ on $\ell$ on the other side of $y$ than $a$. Therefore, to prove our claim, it is sufficient to prove that $y \in \partial A_{a}$ for every $y \in \partial Q \cap A$. Suppose this were not true, without loss of generality suppose there is some point in $\ell_{a, b} \cap Q \cap A$ 
which is not in $\partial A_{a}$. Then, since $m_{a, b, c} \in \partial A_{a}$ and since $A_{a}$ is convex there is a $\widehat{y} \in \ell_{a, b} \cap Q \cap A$ such that $\left[m_{a, b, c}, \widehat{y}\right] \subseteq \partial A_{a}$ and such that $\left(\ell_{a, b} \cap Q\right) \backslash\left[m_{a, b, c}, \widehat{y}\right] \neq \emptyset$ but $\left(\ell_{a, b} \cap Q\right) \backslash\left[m_{a, b, c}, \widehat{y}\right] \cap \bar{A}_{a}=\emptyset$. Then there must be a $z \in \mathcal{B}$ such that $\ell_{a, z}$ intersects $\ell_{a, b}$ at the point $\widehat{y}$. Since, clearly, $\widehat{y} \in \partial A_{a} \cap A^{\circ}$ and condition (ii) in Lemma 3.9 does not hold, there must be a $v \in \mathcal{B}$ such that the set $\{a, z, b, v\}$ is rectangular. This implies in particular that $\ell_{a, z}$ and $\ell_{a, b}$ are perpendicular. But then $y \notin A_{a}$ for all $y$ in $Q^{\circ} \cap A$ on the straight line $\ell^{\prime}$ through $a$ and $\widehat{y}$. This is a contradiction with the observation at the beginning of the proof, since $\widehat{y} \in \partial A_{a}$. This completes the proof of the claim in Step 2.

Step 3 Let $a \in \mathcal{B}$. Then the set $\bar{A}_{a}$ is the intersection of $A$ with an orthant $Q_{a}$ with $p\left(Q_{a}\right) \in A^{\circ}$.

To prove this, just note that by Lemma 3.10 there are $b, c$ as in Step 2. The claim of Step 3 now follows from Step 2.

We can now complete the proof of the only-if direction. By Step 3, for each $a \in \mathcal{B}$ the set $\bar{A}_{a}$ is the intersection of $A$ with an orthant $Q_{a}$ with $p\left(Q_{a}\right) \in A^{\circ}$. Moreover, $A=\bigcup_{a \in \mathcal{B}} \bar{A}_{a}=\bigcup_{a \in \mathcal{B}}\left(Q_{a} \cap A\right)$. Hence by Step $1,|\mathcal{B}|=4$, in particular $\mathcal{B}=\{a, b, c, x\}$ with $a, b, c, x$ as in Step 2. Thus, $\mathcal{B}$ is rectangular.

For the if-direction, let $\mathcal{B}$ be rectangular with $\mathcal{B}=\{v, w, y, z\}$. Clearly, among any three distinct points in $\{v, w, y, z\}$ there is a point $a$ such that $m_{a, b, c} \in \partial A_{a} \cap A^{\circ}$, where $b$ and $c$ are the other two points, but together with the fourth point they form the rectangular set $\mathcal{B}$. Hence, condition (ii) does not hold.

We can now formulate the main result of this section. Call $\varphi$ dictatorial if there is a dictator, i.e., an agent $d \in N$ such that $\varphi(p) \in \mathfrak{b}(p(d))$ for all $p \in A^{N}$.

Theorem 3.12 If $|\mathcal{B}| \geq 3$ and $\mathcal{B}$ is not rectangular then $\varphi$ is dictatorial.

Proof. By Lemmas 3.8, 3.9, and 3.11 the set of decisive coalitions is an ultrafilter. Hence, by Lemma 2.2, there is a $d \in N$ such that $d$ is decisive. So $\varphi$ is dictatorial with dictator $d$.

We conclude this section with two remarks. The first remark is about regular polytopes.

Remark 3.13 Call a polytope $A$ in $\mathbb{R}^{2}$ regular if its extreme points - the elements of the set $C$ - are the vertices of a regular polygon (equilateral triangle, square, etc.). For such a regular polytope Theorem 3.12 implies that $\varphi$ is dictatorial if and only if $|C| \neq 4$. Thus, on regular polytopes $A$, a strategy-proof and Pareto optimal rule is dictatorial unless $A$ is a square including its inside.

The second remark concerns a relation between the results of Gibbard (1973) and Satterthwaite (1975) and Theorem 3.12.

Remark 3.14 Let $C=\{a, b, c\}$ such that $a, b$, and $c$ are the vertices of an acute triangle. By Theorem 3.12 any strategy-proof and Pareto optimal rule 
on $A$ must be a dictatorship. An alternative proof of this fact could work as follows. Let $\varphi$ be such a rule and first prove that $\varphi(p) \in C$ for every $p \in A^{N}$. Then, effectively, $\varphi$ is a strategy-proof and Pareto optimal social choice function on the set $C$ of alternatives, with full domain of preferences, as can be seen in Fig. 5(a), where the perpendicular bisectors of the edges are drawn. Hence, $\varphi$ must be a dictatorship according to the Gibbard-Satterthwaite theorem. If the triangle is not acute then only four strict preferences are generated (Fig. 5(b)) and thus the Gibbard-Satterthwaite theorem does not apply. Indeed, in this case, if the right or obtuse angle is at $c$, then $a$ and $b$ are the only single-best points, and for instance majority voting between $a$ and $b$ is a strategy-proof and Pareto optimal rule.

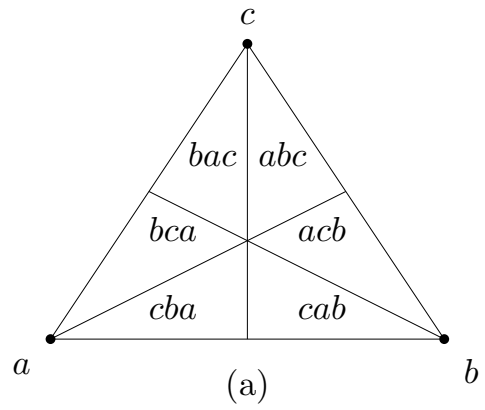

Figure 5: Remark 3.14

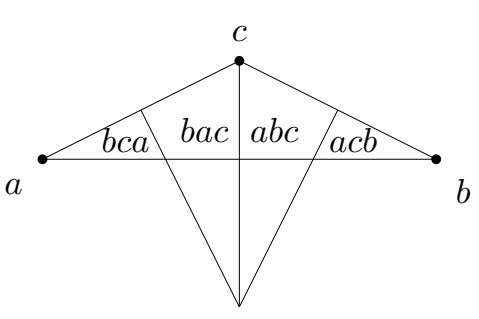

(b)

\section{Nondictatorship}

In this section we study the two cases excluded in Theorem 3.12, namely the case $|\mathcal{B}|=2$ and the case where $\mathcal{B}$ is rectangular.

\section{$4.1|\mathcal{B}|=2$}

According to Theorem 3.12 dictatorship is implied by Pareto optimality and strategy-proofness under two conditions on the set $\mathcal{B}$ of single-best alternatives in $C$. In this section we consider the case where the first one of these conditions does not hold. More precisely, we assume that $|\mathcal{B}|=2$, and write $\mathcal{B}=\{a, b\}$. The following lemma reveals a consequence of this assumption. It says that the center of the circle through $a, b$, and any other point $c \in C$ cannot be an interior point of $A$.

Lemma 4.1 Let $c \in A \backslash\{a, b\}$. Then $m_{a, b, c} \notin A^{\circ}$.

Proof. First observe that for any $B \subseteq C$ with $|B| \geq 2$ we have that $A_{B}$, if not empty, consists of a line segment (which may happen if $|B|=2$ ) or a single 
point. This implies that any open subset of $A^{\circ}$ has a non-empty intersection with $A_{a}$ or $A_{b}$. Now suppose $m_{a, b, c} \in A^{\circ}$. Then the set $\left(V_{c a b}^{\circ} \cup V_{c b a}^{\circ}\right) \cap A^{\circ}$, where $V_{c a b}=\left\{x \in A:(c, a),(a, b) \in R_{x}\right\}$ and $V_{c b a}=\{x \in A:(c, b),(b, a) \in$ $\left.R_{x}\right\}$, is an open subset of $A^{\circ}$. However, $A_{a} \cap\left(V_{c a b}^{\circ} \cup V_{c b a}^{\circ}\right) \cap A^{\circ}=\emptyset$ and $A_{b} \cap\left(V_{c a b}^{\circ} \cup V_{c b a}\right)^{\circ} \cap A^{\circ}=\emptyset$. This is a contradiction, hence $m_{a, b, c} \notin A^{\circ}$.

For the remainder of this section let again $\varphi$ be a Pareto optimal and strategy-proof rule. We first show that in almost all cases we have $\varphi(p) \in\{a, b\}$.

Lemma 4.2 Let $p \in A^{N}, p \neq m_{a, b, c}^{N}$ for all $c \in C \backslash\{a, b\}$. Then $\varphi(p) \in\{a, b\}$.

Proof. To the contrary suppose $\varphi(p) \notin\{a, b\}$. We write $m$ for $m_{a, b, \varphi(p)}$ if $a, b$, and $\varphi(p)$ are not collinear; otherwise, $\varphi(p) \in] a, b[$ and we take $m=\varphi(p)$. (Below it will follow that $\varphi(p) \in \partial A$, so that in view of Lemma 3.1 the latter case will actually not occur.) For every $i \in N$ with $p(i) \notin\{a, b, m\}$ we have by Lemma 4.1 that $\|p(i)-\varphi(p)\|<\|p(i)-a\|$ or $\|p(i)-\varphi(p)\|<\|p(i)-b\|$. If $\|p(i)-\varphi(p)\|<$ $\|p(i)-a\|$ for some $i \in N$, then consider the profile $p^{\prime}$ with $p^{\prime}(i)=b$ and $p^{\prime}(j)=p(j)$ for all $j \neq i$. If $\varphi\left(p^{\prime}\right)=b$ then agent $i$ would manipulate from $p^{\prime}$ to $p$ since $\varphi(p) \neq b$, and if $\varphi\left(p^{\prime}\right)=a$ then agent $i$ would manipulate from $p$ to $p^{\prime}$. Hence, strategy-proofness implies $\varphi\left(p^{\prime}\right) \notin\{a, b\}$. If $\|p(i)-\varphi(p)\|<\|p(i)-b\|$ for some $i \in N$ then we consider the profile $p^{\prime \prime}$ with $p^{\prime \prime}(i)=a$ and $p^{\prime \prime}(j)=p(j)$ for all $j \neq i$ and similarly derive that $\varphi\left(p^{\prime \prime}\right) \notin\{a, b\}$. Therefore we may assume that $p(N) \subseteq\{a, b, m\}$. Since $p(N) \subseteq\{a, b\}$ would contradict Lemma 3.6 we may further assume that $p$ is of the form $p=\left(a^{S}, b^{T}, m^{V}\right)$ for some coalitions $S, T$, and $V=N \backslash(S \cup T) \neq \emptyset$. By Pareto optimality we have that either $S$ and $T$ are non-empty or $V=N$.

Suppose that $\varphi(p) \in A^{\circ}$. Then there are points of $A$ on the straight line through $\varphi(p)$ perpendicular to $\ell(a, b)$ that Pareto dominate $\varphi(p)$ given the profile $p$, contradicting Pareto optimality of $\varphi$. Hence, $\varphi(p) \in \partial A$, so that $\varphi(p) \in C$ by Lemma 3.1. Thus, by the condition on $p$ in the lemma, $V \neq N$, so that $S, T, V \neq \emptyset$.

Next, let $\bar{a}=m_{a, \varphi(p)}$ and consider profile $p^{a}=\left(\bar{a}^{S}, b^{T}, m^{V}\right)$. We prove that $\varphi\left(p^{a}\right)=a$. (See Fig. 6 for an illustration of this part of the argument.) By comparing $p$ and $p^{a}$, strategy-proofness implies that $\left\|\varphi\left(p^{a}\right)-\bar{a}\right\| \geq\|\varphi(p)-\bar{a}\|=$ $\|a-\bar{a}\|$ and $\|\varphi(p)-a\| \geq\left\|\varphi\left(p^{a}\right)-a\right\|$. Consider the profile $q=\left(\bar{a}^{S}, b^{T \cup V}\right)$. Note that $b \in A_{a}$; also, since by Lemma $4.1, m_{a, b, c} \notin A^{\circ}$ and $m_{a, b, c} \in \ell_{a, b}$ for all $c \in A \backslash\{a, b\}$, it follows that $\bar{a} \in A_{b}$. Now Lemma 3.6 implies $\varphi(q) \in\{a, b\}$, hence strategy-proofness yields $\|m-a\|=\|m-b\| \leq\left\|m-\varphi\left(p^{a}\right)\right\|$. This means that $\varphi\left(p^{a}\right)$ is not inside the circle with center $m$ through the points $a, b$ and $\varphi(p)$, but then $\varphi\left(p^{a}\right)$ must be on this circle since there are no points of $A$ outside this circle (since otherwise we could find a point in $\mathcal{B}$ different from $a$ and $b$, a contradiction). Hence, $\|m-a\|=\left\|m-\varphi\left(p^{a}\right)\right\|$. Since $\|\varphi(p)-a\| \geq\left\|\varphi\left(p^{a}\right)-a\right\|$, $\left\|\varphi\left(p^{a}\right)-\bar{a}\right\| \geq\|a-\bar{a}\|$ and $\|m-a\|=\left\|m-\varphi\left(p^{a}\right)\right\|$ it follows that either $\varphi\left(p^{a}\right)=\varphi(p)$ or $\varphi\left(p^{a}\right)$ is on the circle with center $m$ through $a$ and $\varphi(p)$ on the arc between $a$ and $b$ not containing $\varphi(p)$. The latter case, however, implies $\varphi\left(p^{a}\right)=a$ since there cannot be points of $C$ on this arc other than $a$ or $b$. Hence, 


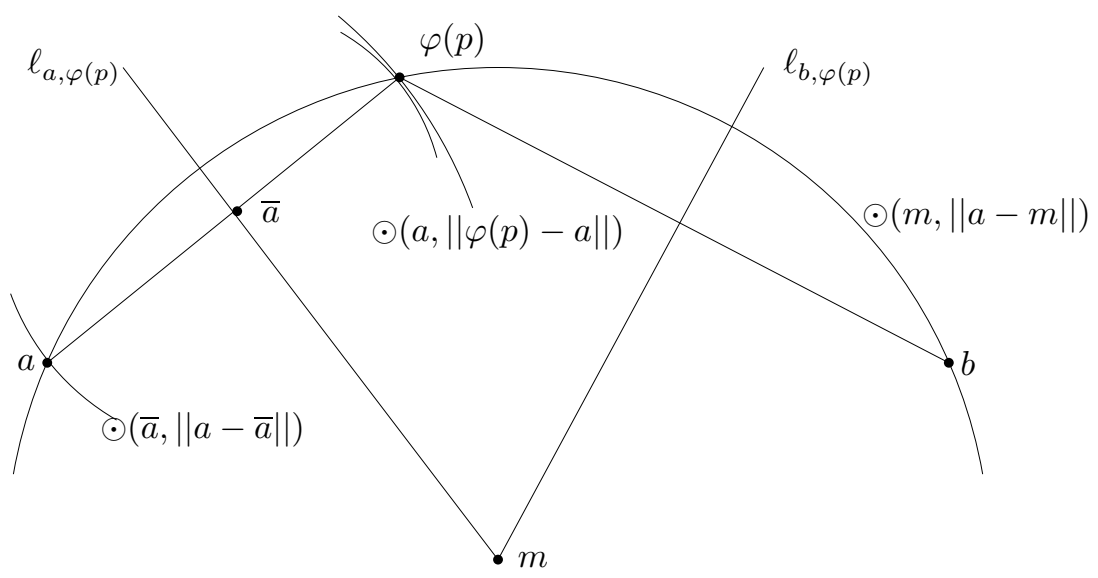

Figure 6: Illustrating the proof of Lemma 4.2

$\varphi\left(p^{a}\right) \in\{a, \varphi(p)\}$. But $\varphi(p)$ is Pareto dominated by $a$ at the profile $p^{a}$. So, $\varphi\left(p^{a}\right)=a$.

Now let $\bar{b}=m_{b, \varphi(p)}$ and consider the profile $p^{a b}=\left(\bar{a}^{S}, \bar{b}^{T}, m^{V}\right)$. Note that $\bar{b} \in \mathcal{H}^{\circ}\left(\ell_{a, b}, b\right)$. Similarly as above we have $\bar{b} \in A_{a}$. Therefore $\varphi\left(p^{a b}\right)=a$ by strategy-proofness.

By an analogous argument but now starting with the profile $p^{b}=$ $\left(a^{S}, \bar{b}^{T}, m^{V}\right)$, we obtain $p^{a b}=b$, a contradiction which completes the proof.

In order to characterize all strategy-proof and Pareto optimal rules while avoiding technicalities due to preference indifferences, we make the following additional assumptions. The first assumption takes care of the cases not covered by Lemma 4.2. More precisely, suppose $m_{a, b, c} \in A$ for some $c \in C \backslash\{a, b\}$. Then by Lemma 4.1 we must have $m_{a, b, c} \in\{\underline{m}, \bar{m}\}$, where $\{\underline{m}, \bar{m}\}=\partial A \cap \ell_{a, b}$.

Assumption $4.3 \varphi(p) \in\{a, b\}$ for $p=\underline{m}^{N}$ and $p=\bar{m}^{N}$.

The second assumption, which is a version of the familiar non-bossiness condition, guarantees that indifferent agents cannot change the assigned alternative.

Assumption 4.4 For all $i \in N$ and $p, q \in A^{N}$ such that $p(j)=q(j)$ for all $j \in N \backslash\{i\}$, if $\|p(i)-a\|=\|p(i)-b\|$ and $\|q(i)-a\|=\|q(i)-b\|$, then $\varphi(p)=\varphi(q)$.

With these additional assumptions, it is straightforward to verify that (a strategy-proof and Pareto optimal rule) $\varphi$ depends only on the individual preferences between $a$ and $b$ and not on the exact location of the dips. We will now develop a precise description of $\varphi$.

Let $\mathcal{W}_{a}^{\varphi}$ be the set of pairs $(S, U) \in N \times N$ with $S \cap U=\emptyset$ and such that $\varphi(p)=a$ for all profiles $p$ with $a$ single-best for all agents in $S$ and both $a$ and $b$ 
best for all agents in $U$. Let $\mathcal{W}_{b}^{\varphi}$ be defined analogously. Since $\varphi$ depends only on the individual preferences between $a$ and $b$ we have that the pair $\left(\mathcal{W}_{a}^{\varphi}, \mathcal{W}_{b}^{\varphi}\right)$ is both proper and strong, i.e., either $(S, U) \in \mathcal{W}_{a}^{\varphi}$ or $(T, U) \in \mathcal{W}_{b}^{\varphi}$ for all pairwise disjoint sets $S, U$ and $T$ with $S \cup T \cup U=N$. Pareto-optimality of $\varphi$ implies that the pair $\left(\mathcal{W}_{a}^{\varphi}, \mathcal{W}_{b}^{\varphi}\right)$ is Pareto optimal, i.e., $(S, U) \in \mathcal{W}_{a}^{\varphi}$ if $S \neq \emptyset$ and $S \cup U=N$, and $(T, U) \in \mathcal{W}_{b}^{\varphi}$ if $T \neq \emptyset$ and $T \cup U=N$. Furthermore, strategy-proofness of $\varphi$ implies that the pair $\left(\mathcal{W}_{a}^{\varphi}, \mathcal{W}_{b}^{\varphi}\right)$ is monotone, i.e., $\left(S^{\prime}, U^{\prime}\right) \in \mathcal{W}_{a}^{\varphi}$ whenever $(S, U) \in \mathcal{W}_{a}^{\varphi}$ and $S \subseteq S^{\prime}$ and $S \cup U \subseteq S^{\prime} \cup U^{\prime}$ and $\left(T^{\prime}, U^{\prime}\right) \in \mathcal{W}_{b}^{\varphi}$ whenever $(T, U) \in \mathcal{W}_{b}^{\varphi}$ and $T \subseteq T^{\prime}$ and $T \cup U \subseteq T^{\prime} \cup U^{\prime}$. It is straightforward to check that any proper and strong, Pareto optimal and monotone pair $\left(\mathcal{W}_{a}, \mathcal{W}_{b}\right)$ defines a Pareto optimal and strategy-proof rule $\varphi$, satisfying Assumptions 4.3 and 4.4, with $\left(\mathcal{W}_{a}^{\varphi}, \mathcal{W}_{b}^{\varphi}\right)=\left(\mathcal{W}_{a}, \mathcal{W}_{b}\right)$. We summarize these findings in the following theorem.

Theorem 4.5 Let $\mathcal{B}=\{a, b\}$ with $a \neq b$. Then

(i) If $\varphi$ is strategy-proof, Pareto optimal, and satisfies Assumptions 4.3 and 4.4 , then $\left(\mathcal{W}_{a}^{\varphi}, \mathcal{W}_{b}^{\varphi}\right)$ is proper and strong, Pareto optimal and monotone.

(ii) If $\left(\mathcal{W}_{a}, \mathcal{W}_{b}\right)$ is proper and strong, Pareto optimal and monotone, then there $i s$ a strategy-proof, and Pareto optimal rule $\varphi$, satisfying Assumptions 4.3 and 4.4 , such that $\mathcal{W}_{a}=\mathcal{W}_{a}^{\varphi}$ and $\mathcal{W}_{b}=\mathcal{W}_{b}^{\varphi}$.

\section{2 $\mathcal{B}$ is rectangular}

In this section we consider the case where $\mathcal{B}$ is rectangular. Thus, we assume that $\mathcal{B}=\{a, b, c, d\}$ and that $[a, b],[b, c],[c, d]$, and $[d, a]$ are the edges of a rectangle. Rather than attempting to describe all strategy-proof and Pareto efficient rules for this case - which becomes a very technical exercise mainly due to many tie-breaking decisions that have to be made - we content ourselves with giving a typical example of a rule. ${ }^{5}$

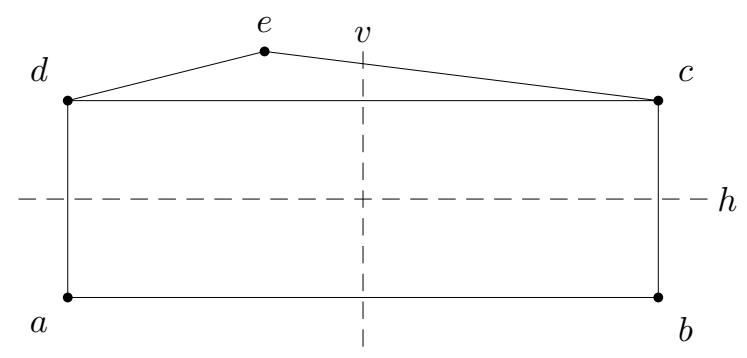

Figure 7: Illustrating Example 4.6

\footnotetext{
${ }^{5} \mathrm{An}$ early draft of this paper contains a complete characterization of all such rules under some additional assumptions.
} 
Example 4.6 (Cf. Fig 7.) Let $h$ be the common perpendicular bisector of $[a, d]$ and $[b, c]$ and let $v$ be the common perpendicular bisector of $[a, b]$ and $[c, d]$. For every profile $p$ define $\varphi(p)$ as follows. If $|\{i \in N: p(i) \in H(h, a)\}| \geq \mid\{i \in N$ : $p(i) \in H(h, d)\} \mid$ then $\varphi(p) \in\{c, d\}$ and if $|\{i \in N: p(i) \in H(h, a)\}|<\mid\{i \in$ $N: p(i) \in H(h, d)\} \mid$ then $\varphi(p) \in\{a, b\}$. If $|\{i \in N: p(i) \in H(v, a)\}| \geq \mid\{i \in$ $N: p(i) \in H(v, b)\} \mid$ then $\varphi(p) \in\{b, c\}$ and if $|\{i \in N: p(i) \in H(v, a)\}|<\mid\{i \in$ $N: p(i) \in H(v, b)\} \mid$ then $\varphi(p) \in\{a, d\}$. In words, we let majority voting decide between $\{a, b\}$ and $\{c, d\}$ on the one hand, and between $\{a, d\}$ and $\{b, c\}$ on the other. It can be proved (left to the reader) that this rule is strategy-proof and Pareto optimal.

\section{Concluding remarks}

The preceding sections naturally lead to the question if the obtained results can be extended to other regions, such as general compact convex sets. Since some of the proofs seem to rely heavily on the finiteness of the set of extreme points, this question does not appear to have a straightforward answer. In a companion note (Öztürk, Peters, and Storcken, 2012) we show that the dictatorship result extends to the disc (circle and its inside) in $\mathbb{R}^{2}$. This result may not come as a surprise in view of the fact the the disc can be seen as the 'limit' of polytopes for which Theorem 3.12 implies dictatorship, but nevertheless we need a separate proof to show this.

Another potential extension concerns the domain of preferences. One might expect, however, that allowing for a richer class of single-dipped preferences, other than only Euclidean, is bound to result in dictatorship.

\section{References}

Barbera S, Berga D, Moreno B (2009) Single-dipped preferences. Barcelona Economics Working Paper Series No 418

Besfamille M, Lozachmeur JM (2010) NIMBY and mechanism design under different constitutional constraints. International Tax and Public Finance, 17:114-132

Black D (1948) On the rationale of group decision-making. The Journal of Political Economy 56:23-34

Border KC, Jordan JS (1983) Straightforward elections, unanimity and phantom voters. Review of Economic Studies 50:153-170

Ehlers L (2002) Probabilistic allocation rules and single-dipped preferences. Social Choice and Welfare 19:325-348

Gibbard A (1973) Manipulation of voting schemes: a general result. Econometrica 41:587-602

Hansson B (1976) The existence of group preference functions. Public Choice 38, 89-98

Inada K (1964) A note on the simple majority rule. Econometrica 31:525-531 
Kirman A, Sondermann D (1972) Arrow's theorem, many agents, and invisible dictators. Journal of Economic Theory 3, 267-277

Klaus B (2001) Coalitional strategy-proofness in economies with single-dipped preferences and the assignment of an individual object. Games and Economic Behavior $34,64-82$

Klaus B, Peters H, Storcken T (1997) Strategy-proof division of a private good when preferences are single-dipped. Economic Letters 55:339-346

Kunreuther H, Kleindorfer PR (1986). A sealed-bid auction mechanism for siting noxious facilities. American Economic Review (Papers and Proceedings) 76:295299

Lescop D (2007) Optimal mechanisms for siting noxious facilities. Review of Economic Design 10:273-284

Manjunath V (2009) Efficient and strategy-proof social choice when preferences are single-dipped. Working paper

Moulin H (1980) On strategy-proofness and single peakedness. Public Choice 35:437455

Öztürk M, Peters H, Storcken T (2012) Strategy-proof location of a public bad on a disc. Research Memorandum, Maastricht

Peremans W, Storcken T (1999) Strategy-proofness on single-dipped preference domains. In de Swart HMC (ed.) Logic, Game theory and Social choice. Tilburg University Press, The Netherlands

Satterthwaite MA (1975) Strategy-proofness and Arrow's conditions: existence and correspondence theorem for voting procedures and social choice functions. Journal of Economic Theory 10:187-217 